University of Wollongong

Research Online

Faculty of Engineering and Information

Faculty of Engineering and Information

Sciences - Papers: Part A

Sciences

$1-1-2015$

\title{
Simulink model for examining dynamic interactions involving electro- mechanical oscillations in distribution systems
}

Dothinka Ranamuka Rallage

University of Wollongong, dssrr987@uowmail.edu.au

Ashish P. Agalgaonkar

University of Wollongong, ashish@uow.edu.au

Kashem M. Muttaqi

University of Wollongong, kashem@uow.edu.au

Follow this and additional works at: https://ro.uow.edu.au/eispapers

Part of the Engineering Commons, and the Science and Technology Studies Commons

Research Online is the open access institutional repository for the University of Wollongong. For further information contact the UOW Library: research-pubs@uow.edu.au 


\title{
Simulink model for examining dynamic interactions involving electro-mechanical oscillations in distribution systems
}

\begin{abstract}
In Australian medium voltage (MV) distribution networks, the majority of embedded local generation (LG) uses synchronous machine based technology. LG units with a synchronous generator can easily be dispatched and controlled to provide or absorb reactive power and thereby locally supporting the system voltage and increasing the voltage stability margin. The fast response from a synchronous machine based LG unit exciter will ensure fast voltage recovery. This will significantly improve the transient voltage stability. Also, the synchronous machine based LG units can provide inertial support to the system.

Furthermore, some of these LG technologies work as standby power supplies all over the world. However, there can be dynamic interactions between nearby synchronous machine based LG units leading to significant rotor swings. It is mainly because of (a) low inertia coefficient associated with the machines and (b) the operation of excitation system deteriorates the damping of oscillations that follow the first rotor swing after the perturbation where a fast responding excitation system can reduce the damping torque component. This paper demonstrates modelling MV distribution systems using MATLAB-SimuLink and conventional modelling technique for investigating inter-unit electro-mechanical oscillations in nearby LG units.
\end{abstract}

\section{Keywords}

oscillations, mechanical, electro, involving, interactions, dynamic, examining, model, systems, simulink, distribution

Disciplines

Engineering | Science and Technology Studies

\section{Publication Details}

D. Ranamuka , A. P. Agalgaonkar \& K. M. Muttaqi, "Simulink model for examining dynamic interactions involving electro-mechanical oscillations in distribution systems," in Power Engineering Conference (AUPEC), 2015 Australasian Universities, 2015, pp. 1-6. 


\title{
Simulink Model for Examining Dynamic Interactions Involving Electro-Mechanical Oscillations in Distribution Systems
}

\author{
D. Ranamuka, Student Member, IEEE, A. P. Agalgaonkar, Senior Member, IEEE, K. M. Muttaqi, \\ Senior Member, IEEE
}

\begin{abstract}
In Australian medium voltage (MV) distribution networks, the majority of embedded local generation (LG) uses synchronous machine based technology. LG units with a synchronous generator can easily be dispatched and controlled to provide or absorb reactive power and thereby locally supporting the system voltage and increasing the voltage stability margin. The fast response from a synchronous machine based LG unit exciter will ensure fast voltage recovery. This will significantly improve the transient voltage stability. Also, the synchronous machine based LG units can provide inertial support to the system. Furthermore, some of these LG technologies work as standby power supplies all over the world. However, there can be dynamic interactions between nearby synchronous machine based LG units leading to significant rotor swings. It is mainly because of (a) low inertia coefficient associated with the machines and (b) the operation of excitation system deteriorates the damping of oscillations that follow the first rotor swing after the perturbation where a fast responding excitation system can reduce the damping torque component. This paper demonstrates modelling MV distribution systems using MATLAB-SimuLink and conventional modelling technique for investigating inter-unit electro-mechanical oscillations in nearby LG units.
\end{abstract}

Index Terms--distribution systems; electro-mechanical oscillations; interactions; local generation.

\section{NOMENCLATURE}

$\begin{array}{lll}\text { MV } & \text { Medium voltage } & \omega_{b} \\ \text { LG } & \text { Local generation } & \\ F_{1} & \text { Stator magnetomotive force } & T_{\text {mech }} \\ F_{2} & \text { Rotor magnetomotive force } & T_{\text {damp }} \\ \Psi & \text { Resultant of the vector addition of } F_{1} \text { and } F_{2} & \delta \\ a, b, c & \text { Phase- } a, b \text { and } c \text { of a three phase system } & \omega_{r m} \\ v_{q} & \text { Stator voltage on rotor } q \text { do-reference frame } & P \\ & \text { (q-axis component) } & Y_{\text {system }} \\ r_{s} & \text { Stator winding resistance } & n\end{array}$

$r_{s} \quad$ Stator winding resistance

$i_{q} \quad$ Stator winding current on rotor qdo-reference frame (q-axis component)

$x^{\prime}{ }_{d} \quad$ Sub-transient reactance (d-axis component)

$i_{d} \quad$ Stator winding current on rotor qdo-reference frame (d-axis component)

The authors are with the Australian Power Quality and Reliability Centre, School of Electrical, Computer and Telecommunications Engineering, University of Wollongong, New South Wales 2522 Australia. (e-mail: dssrr987@uowmail.edu.au; ashish@uow.edu.au; kashem@uow.edu.au)

\begin{tabular}{|c|c|}
\hline$E_{q}^{\prime}$ & $\begin{array}{l}\text { Voltage behind the sub-transient inductance ( } q \text { - } \\
\text { axis component) }\end{array}$ \\
\hline$v_{d}$ & $\begin{array}{l}\text { Stator voltage on rotor qdo-reference frame ( } d \text { - } \\
\text { axis component) }\end{array}$ \\
\hline$x^{\prime}{ }_{q}$ & Sub-transient reactance (q-axis component) \\
\hline$E^{\prime}{ }_{d}$ & $\begin{array}{l}\text { Voltage behind the sub-transient inductance ( } d \text { - } \\
\text { axis component) }\end{array}$ \\
\hline$\lambda_{q}^{\prime}$ & Sub-transient flux linkage ( $d$-axis component) \\
\hline$\lambda_{q}$ & Steady state flux linkage ( $q$-axis component) \\
\hline$L_{q}^{\prime}$ & Sub-transient inductance ( $q$-axis component) \\
\hline$\lambda_{d}^{\prime}$ & Sub-transient flux linkage ( $d$-axis component) \\
\hline$\lambda_{d}$ & Steady state flux linkage (d-axis component) \\
\hline$L_{d}^{\prime}$ & Sub-transient inductance ( $d$-axis component) \\
\hline$\omega_{e}$ & Synchronous speed \\
\hline$T^{\prime}{ }_{d o}$ & $\begin{array}{l}\text { Sub-transients time constant } \\
\text { component) }\end{array}$ \\
\hline$E_{f}$ & Field winding voltage \\
\hline$x_{d}$ & Steady state reactance ( $d$-axis component) \\
\hline$T_{q o}^{\prime}$ & $\begin{array}{l}\text { Sub-transients time constant } \quad(d \text {-axis } \\
\text { component) }\end{array}$ \\
\hline$x_{q}$ & Steady state reactance ( $q$-axis component) \\
\hline$T_{e m}$ & Electro-mechanical torque \\
\hline$H$ & Inertia coefficient \\
\hline$\omega_{r}$ & Rotor speed \\
\hline$\omega_{b}$ & $\begin{array}{l}\text { Base value for speed per unitization where } f_{b} \text { is } \\
\text { the electrical frequency of the system }\end{array}$ \\
\hline$T_{\text {mech }}$ & Mechanical torque \\
\hline$T_{\text {damp }}$ & Frictional and windage torque \\
\hline$\delta$ & Rotor angle \\
\hline$\omega_{r m}$ & Prime mover speed \\
\hline$P$ & Number of poles \\
\hline$Y_{\text {system }}$ & Admittance matrix of the distribution system \\
\hline$n$ & Number of buses in the distribution system \\
\hline
\end{tabular}

\section{INTRODUCTION}

T $\mathrm{N}$ the medium voltage active distribution networks, the $\mathbf{1}_{\text {small to medium scale synchronous machine based local }}$ generators which normally have low inertia coefficient are primarily utilized for active voltage control (normal-sate) in the system enabling an excitation system [1]. However, it may lead to not only the interactions among local generation units and voltage regulating devices; but also interactions among the nearby local generation units [1]-[4]. Therefore, it is necessary 
to investigate and analyse those interactions and their impact on the system electric variables in order to determine whether any supplementary control is required or not.

The electrical power generated by a synchronous generator is equal to the mechanical power applied by the prime mover, when the associated losses are assumed zero. In the steady state, output electrical power balances the input mechanical power. Also, the associated mechanical torque is in the direction of rotor rotation, while the electrical torque (comprising synchronising torque component dealing with machine synchronism and damping torque component dealing with damping rotor oscillations) is applied to the shaft by the generator is in a direction opposite to the rotor rotation. If the generator system is perturbed, the output electrical power of the generator can change rapidly, while the input mechanical power is relatively slow to change. Due to this difference in response times, there can be temporary unbalance between output electrical power and input mechanical power. Due to this power unbalance, there can be a difference in torque applied to the shaft leading to rotor acceleration or deceleration. When the rotor speed is changed, the relative rotor angle is changed causing rotor swings and instability [5]. A fast excitation system aids to eliminate transient instability within the first swing following large perturbations by managing a sufficient synchronizing torque component. However, the operation of excitation system deteriorates the damping of oscillations that follow the first rotor swing after the perturbation; because a fast responding excitation system can reduce the damping torque component. Therefore, it is clear that the operation of an excitation system can significantly contribute to small signal instability of a power system, especially where low inertia machines are dominant because the low values of inertia coefficient will increase the values of both natural frequency of oscillations and damping coefficient. This is the motivation for the work associated with this paper.

This paper is based on dynamic modelling of synchronous machine based LG units and MV distribution systems for such interaction studies using MATLAB-SimuLink and conventional modelling technique. The main interest is for transients of the electro-mechanical oscillations among the local generation units, where round rotor machines are common in MV distribution level. Using this model, the impact of machine inertia, excitation system and its tuned parameters, on-line adjustment in voltage reference value for excitation system and network strength on inter-unit oscillations can be investigated and analyzed. Modelling using MATLAB-SimuLink helps design supplementary control when required, since MATLAB is rich with variety of control tool boxes (such as robust-control tool box), which can easily be linked to the SimuLink.

The paper is organised as follows. Section II details modeling active distribution systems for the interaction studies using MATLAB-SimuLink; and Section III contains a case study, which demonstrates the modeling aspects and possible electro-mechanical oscillations among synchronous machine based LG units providing primary voltage control in normalstate. The Section IV includes the concluding remarks.

\section{MODELING DisTRIBUTION SYSTEMS WITH LOCAL GENERATION USING MATLAB-SIMULINK}

The detail modelling of electric power systems and machines using MATLAB-SimuLink presented in [6]. Since, the main interest is for transients of the electro-mechanical oscillations and the associated interactions, the fast electromagnetic transients are neglected. Also, the representation of network retains only a subset of the network buses where the generation and load behaviour are of interest, hence overall modelling task is based on integrating the conventional transient model for embedded synchronous generators into the localised static network model. In this case, the simplified transient model is used for the embedded synchronous generators in which the changes in stator $q$ - $d$ flux linkages are neglected. This approach enhances the modelling task, especially in case of distribution systems with large set of electric variables which lead to inherently varying operating points resulting into more complex simulations. The overall modelling task includes (i) dynamic modelling of synchronous generators using the transient model, (ii) detail modelling of excitation system and (iii) expressing all voltages and currents in a common reference frame (i.e., synchronously rotating reference frame) and writing all the network equations. While modelling synchronous generators during the transient period the damper windings can be assumed to be no longer active, where overall mathematical model includes (i) stator winding equations, (ii) rotor winding equations, (iii) torque equation and (iv) rotor motion equation as detailed in the following sections.

Fig. 1 shows a diagrammatic representation of rotor angle and stator magnetomotive force, $F_{1}$ and the rotor magnetomotive force, $F_{2}$. By fundamental definition, the rotor angle is the angle between $F_{2}$ and the resultant of the vector addition of $F_{1}$ and $F_{2}, \psi$ [5].

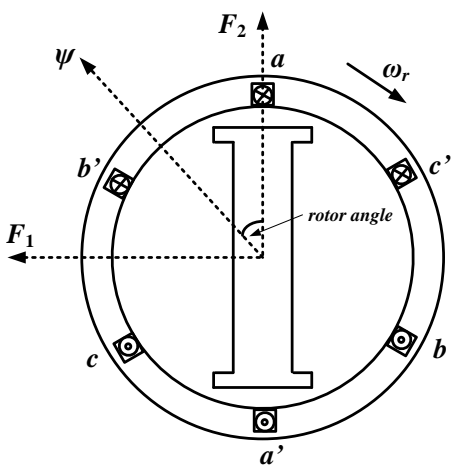

Fig. 1. Diagrammatic representation of rotor angle.

Over the years, experience on power system simulation has shown that the most synchronous generators can adequately be represented by a model that is based on an equivalent idealised machine with one or two sets of damper windings, besides the field winding [7]. Damper windings in the equivalent machine model can be used to represent physical armortisseur windings 
or the damping effects of eddy currents in the solid iron portion of the rotor poles. Amortisseur windings are bars which are found in the rotor of synchronous machines. These bars are short circuited similar to the rotor windings in a squirrel cage machine. The function of these windings is to dampen the torsional oscillations in the rotor that may occur as a result of load fluctuations. They are also known as damper windings. A general circuit representation of idealised machine model of the synchronous machine is shown in Fig. 2. It is noted that the changes in stator flux linkages i.e., the time derivative terms in the stator voltage equations are usually small in comparison to the voltage behind transient inductances. Therefore, those terms in the stator voltage equations can be omitted.

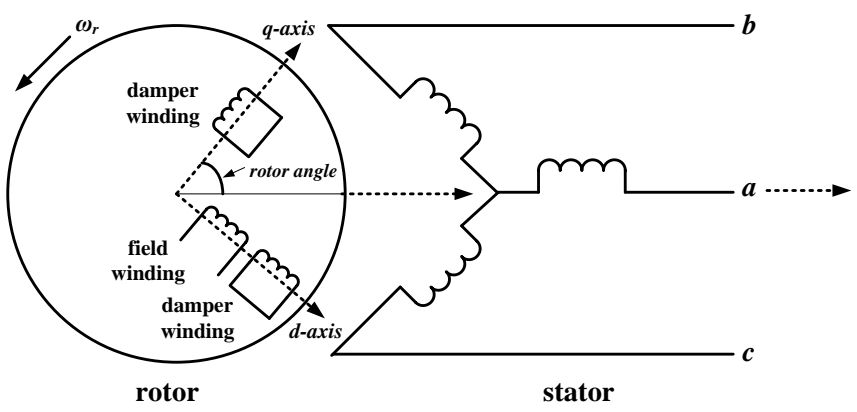

Fig. 2. Diagrammatic circuit representation of idealised machine model of the synchronous machine.

The parameters in the following equations of the synchronous generator (machine) are on per unit basis.

\section{A. Stator Winding Equations}

$v_{q}=-r_{s} \cdot i_{q}-x_{d}^{\prime} \cdot i_{d}+E_{q}^{\prime}$

$v_{d}=-r_{s} \cdot i_{d}+x_{q}^{\prime} \cdot i_{q}+E_{d}^{\prime}$

$\lambda_{q}^{\prime}=\lambda_{q}-L_{q}^{\prime} \cdot\left(-i_{q}\right), \quad \lambda_{d}^{\prime}=\lambda_{d}-L_{d}^{\prime} \cdot\left(-i_{d}\right)$

$E_{d}^{\prime}=-\omega_{e} \cdot \lambda_{q}^{\prime}, \quad E_{q}^{\prime}=\omega_{e} \cdot \lambda_{d}^{\prime}$

\section{B. Rotor Winding Equations}

$T_{d o}^{\prime} \cdot \frac{d}{d t}\left(E_{q}^{\prime}\right)+E_{q}^{\prime}=E_{f}-\left(x_{d}-x_{d}^{\prime}\right) i_{d}$

$T_{q o}^{\prime} \cdot \frac{d}{d t}\left(E_{d}^{\prime}\right)+E_{d}^{\prime}=\left(x_{q}-x_{q}^{\prime}\right) i_{q}$

\section{Torque Equation}

$T_{e m}=-\left\{E_{q}^{\prime} \cdot i_{q}+E_{d}^{\prime} \cdot i_{d}+\left(x_{q}^{\prime}-x_{d}^{\prime}\right) \cdot i_{d} \cdot i_{q}\right\}$

\section{Rotor Motion Equation}

$$
\begin{aligned}
& 2 H \cdot \frac{d\left(\omega_{r}-\omega_{e}\right) / \omega_{b}}{d t}=T_{e m}+T_{\text {mech }}-T_{\text {damp }} \\
& \frac{d \delta_{e}}{d t}=\left(\omega_{r}-\omega_{e}\right), \quad \omega_{\mathrm{r}}=\frac{P}{2} \cdot \omega_{r m}
\end{aligned}
$$

\section{E. Modelling Excitation System}

An accurate modelling of an excitation system as depicted in [8], [9] is essential. Most of the modern excitation systems are of the ac-rotary or ac-static type. Compared to ac-rotary type exciters, the ac-static excitation systems are more compact and have much quicker response time. Most ac-static type exciters draw their primary power from a local ac bus and use controlled rectification to provide an adjustable dc excitation to the field winding of the synchronous generator. Such bus fed systems are dependent on the availability of the ac voltage, which could be adversely affected by nearby faults. There are number of stability issues in which the excitation system plays an important role. On the other hand, the wide variety of design arrangements of excitation systems makes the task of defining generic models difficult. However, mathematical expressions associated with principal components of all excitation systems can be adequate to represent the characteristics of an excitation system. The armature winding usually has a small number of turns compared to the field winding as such; the small resistance and inductance of the armature winding are often neglected. Since, some intended application of these computer models includes large signal variations, limits and non-linearities are considered as important characteristics to be modelled. Also, the output voltage of the exciter is typically a non-linear function of the field and armature currents. In case of saturation, typically, two values of the saturation function are given. Using those two values, a useful region of the saturation curve about the normal operating condition (i.e., operating point) can be approximated by an exponential function. The value of exciter output voltage that produces rated open circuit stator voltage on its air-gap line is chosen as the voltage base. Accordingly, the base value for the exciter field current is calculated. Then, with this per unit system, the exciter's field current and voltage equations are re-written.

\section{F. Modeling Distribution Network with LG units}

A typical MV distribution system with synchronous machine based LG units is shown in Fig. 3.

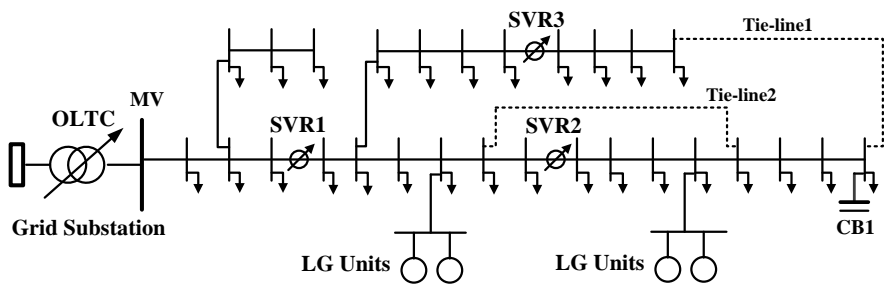

Fig. 3. Representative MV distribution system topology with LG units.

In modelling the test network, voltage phasor angle of infinite bus is conventionally chosen as the reference phasor for the rotor angles and angles of other bus voltages. Next, the time derivative terms of the stator flux linkages are omitted from the stator voltage equations by simplifying the respective transient models of the synchronous generators.The resultant stator voltage equations will be algebraic in nature. Hence, 
algebraic loops will be originated, if the algebraic stator equations and those of the static network model are to be solved separately. In order to avoid algebraic loops involving the network equations and the equations of the stator, all respective equations ought to be properly combined. An algebraic loop in a Simulink model occurs when a signal loop exists with only direct feedthrough blocks within the loop. Algebraic loops are further discussed in the Simulink User's Guide [10]. In order to avoid such algebraic loops from computational perspective; combinatorial equations need to be written. Therefore the stator voltage equations need to be transformed into the synchronously rotating reference frame whose $q$-axis is aligned with the reference phasor as given by (05) and (06), where the transformed terms are now denoted with additional prefix, $e$ [6].

However, the transformation of the stator equations in the rotor reference frame to the synchronously rotating frame of the network involves the time varying rotor angle. If the effective stator impedances in the $q$-axis and $d$-axis are the same, in other words there is no rotor saliency; the resulting impedance in the synchronously rotating frame will not be a function of the time varying rotor angle. The sub-transient saliency of many machines is usually very small. Moreover, where simpler models are desirable, the transient saliency can also be neglected.

$$
\begin{aligned}
& v_{q}-j v_{d}=-r_{s}\left(i_{q}-j i_{d}\right)-x_{d}^{\prime} \cdot i_{d}-j x_{q}^{\prime} \cdot i_{q}+\left(E_{q}^{\prime}-j E_{d}^{\prime}\right) \\
& v_{q}-j v_{d}=e^{-j \delta\left(v_{q}^{e}-j v_{d}^{e}\right), \quad\left(i_{q}-j i_{d}\right)=e^{-j \delta}\left(i_{q}^{e}-j i_{d}^{e}\right)} \\
& v_{q}^{e}-j v_{d}^{e}=-\left(i_{q}^{e}-j i_{d}^{e}\right)-x_{d}^{\prime} \cdot i_{d}^{e}-j x_{q}^{\prime} \cdot i_{q}^{e}+e^{j \delta}\left(E_{q}^{\prime}-j E_{d}^{\prime}\right)
\end{aligned}
$$

If transient saliency is ignored, incorporating the stator voltage equations into the static network model will be straightforward as given by (06).

$$
v_{q}^{e}-j v_{d}^{e}=-\left(r_{s}+x_{d}^{\prime}\right)\left(i_{q}^{e}-j i i_{d}^{e}\right)+e^{j \delta}\left(E_{q}^{\prime}-j E_{d}^{\prime}\right)
$$

Equation (06) can be modelled using a Thevenin's equivalent as shown in Fig. 4. Next, the bus admittance matrix including the transient impedances of the generators, $Y_{\text {system }}$ is derived as given by (07), where total number of buses equal to $n$.

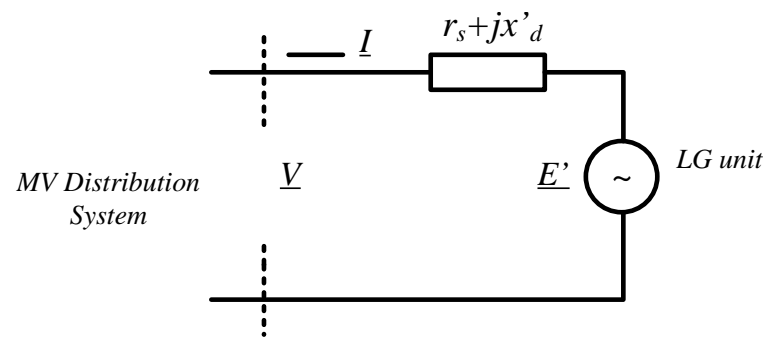

Fig. 4. Thevenin’s equivalent circuit for machine transient model.

$$
\begin{aligned}
& \left(\begin{array}{c}
i_{q}^{e}-j i_{d}^{e} \rightarrow \text { generator bus } \\
\hdashline i_{q}^{e}-j i i_{d}^{e} \rightarrow \text { network bus }
\end{array}\right)_{n \times 1}=\left(Y_{\text {system }}\right)_{n \times n} \times \\
& \times\left(\begin{array}{c}
E_{q}^{\prime}-j E_{d}^{\prime} \rightarrow \text { generator bus } \\
\hdashline v_{q}^{e}-j v_{d}^{e} \rightarrow \text { network bus }
\end{array}\right)_{n \times 1}
\end{aligned}
$$

If the injected current are subjected to load or fault currents; accordingly the corresponding rows and columns in $Y_{\text {system }}$ matrix should be gyrated. Finally, the set of equations in complex variables are rewritten as a set of equations of real variables with twice the order in the form as given by (08) [6]. For large MV power systems, network reduction methods can appropriately be adopted for minimizing the computational burden.

$$
\begin{aligned}
& \left(A_{1}-j A_{2}\right)_{n \times 1}=(G+j B)_{n \times n} \times\left(A_{3}-j A_{4}\right)_{n \times 1} \\
& \left(\begin{array}{c}
A_{1} \\
\hdashline A_{2}
\end{array}\right)_{2 n \times 1}=\left(\begin{array}{c:c}
G & B \\
\hdashline B & G
\end{array}\right)_{2 n \times 2 n} \times\left(\begin{array}{c}
A_{3} \\
\hdashline A_{4}
\end{array}\right)_{2 n \times 1}
\end{aligned}
$$

\section{G. Overall Model in MATLAB-SimuLink}

The transient models of synchronous generators are implemented in MATLAB-SimuLink maintaining initialisation parameters in order to minimise the start-up transients. For the excitation system model, integrators instead of transfer function blocks are used to model the components. It allows initialising the states of excitation system with the outputs from trim function to keep the start-tup transients of the simulation a minimum level. The output from trim function can be used as initial values to get a quick simulation start at the desired operating point. The distribution system equations given by (08) are implemented using matrix gain MATLABSimuLink blocks. Outputs of the network block are the injected currents from the generators. For distribution systems with many nodes, application of per unit system would be beneficial. Then, the parameters of the generators have also to be expressed using the per unit system, appropriately. Finally, the overall SimuLink model which comprises the static network model and transient models of the LG units including excitation system models is appropriately simulated for investigating the interactions between the LG units operated in voltage control mode.

A MATLAB script is used for loading system parameters, setting up different case-study conditions, carrying out modal analysis and plotting results. Eigen values can be derived from the SimuLink model by defining the respective inputs and outputs with inport/outport blocks in place of the source/sink blocks, linmod and eig functions. The participation factors required for model analysis can simply be derived using a MATLAB Code. The model analyses are not detailed in this paper. 


\section{CASE STUDY}

The test system topology shown in Fig. 5 is used for demonstrative simulation case study in this paper. Similar topological combinations can frequently be seen in Australian MV distribution networks, for example in coal mine areas where coal seam gases are used to generate power locally in nearby coal mine sites. For the excitation system model, IEEE Type-1 excitation system is used. The data proposed in [9] for small local generator units and their controls are adopted for the simulation case studies. The simulated system data are shown in Table I, where mechanical torque of the generator, LG1 is varied in small steps (of 0.1 pu) up-down-up at $t=7.5$ s, $15 \mathrm{~s}$ and $22.5 \mathrm{~s}$, respectively for generating the small perturbations. The simulated rated capacity of LG1 and LG2 are 1.5 MVA and 1.0 MVA, respectively.

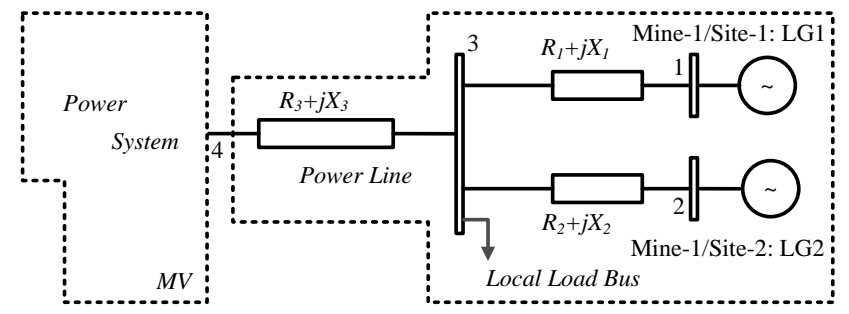

Fig. 5. Test system topology.

TABLE I

\begin{tabular}{c|c|c|c}
\multicolumn{5}{c}{ SIMULATED TEST SYSTEM DATA } \\
\hline $\begin{array}{c}\text { Line: } 1-3 \\
\text { Impedance } \\
/(\mathrm{pu})\end{array}$ & $\begin{array}{c}\text { Line: } 2-3 \\
\text { Impedance }\end{array}$ & $\begin{array}{c}\text { Line: } 3-4 \\
\text { Impedance } \\
/(\mathrm{pu})\end{array}$ & $\begin{array}{c}\text { Local Load } \\
\text { Admittance } \\
/(\mathrm{pu})\end{array}$ \\
\hline $0.004+j 0.1$ & $0.004+j 0.1$ & $0.0015+j 0.08$ & $1.2-j 0.6$ \\
\hline
\end{tabular}

The following case study shows the possibility of occurring inherent oscillations among LG units in distribution systems, a worst case, when the LG units are also utilized for primary voltage control enabling an excitation system. The Fig. 6 (a) shows the rotor angle swings between LG1 and LG2, where Fig. 6 (b) shows the terminal voltage of each LG unit controlled by the excitation system.

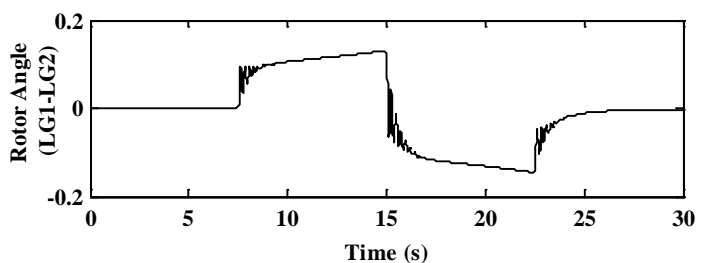

(a)

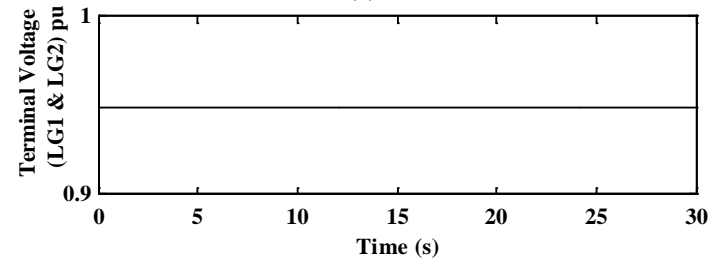

(b)

Fig. 6 (a) Rotor angle swings between LG1 and LG2 units, and (b) Terminal voltage of LG1 and LG2 units.
The variation of rotor angle of LG1 unit is shown Fig. 7.

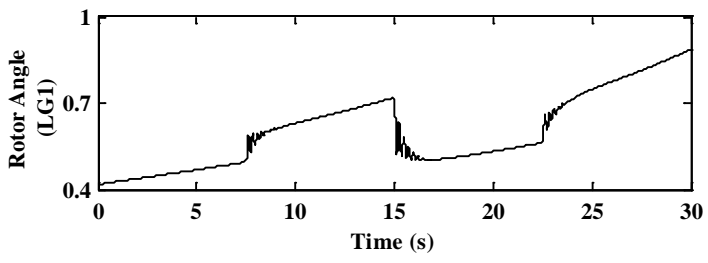

Fig. 7 Rotor angle variation of LG1 unit.

The variation of rotor angle of LG2 unit is shown Fig. 8.

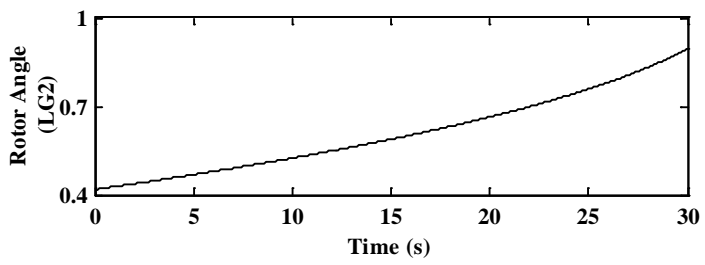

Fig. 8 Rotor angle variation of LG2 unit.

\section{CONCLUSION}

In this paper, dynamic modelling of synchronous machine based LG units and MV distribution systems for interaction studies involving electro-mechanical oscillations are detailed. The MATLAB-SimuLink is used for developing and simulating the models. The motivation behind this work is to observe the respective interactions in case of the low inertia LG units which are also utilized for primary voltage control (normal-sate) in the distribution systems enabling an excitation system. The overall modelling task is based on integrating the conventional transient model for LG units into the localised static network model. This approach enhances the distribution system modelling task, since distribution systems are normally subjected to large set of electric variables which lead to inherently varying operating points causing simulations more complex. The impact of machine inertia, excitation system and its tuned parameters and network strength on inter-unit oscillations can be investigated in detail using this model. The demonstrative test simulation case study reveals that there can be interactions among synchronous machine based LG units which are utilized for primary voltage control in the distribution systems, leading to significant electro-mechanical oscillations.

\section{REFERENCES}

[1] D. Ranamuka, A. P. Agalgaonkar, and K. M. Muttaqi, “On-line Voltage Control in Distribution Systems with Multiple Voltage Regulating Devices,” IEEE Trans. Sustainable Energy, vol. 5, no. 2, pp. 617-628, Apr. 2014.

[2] D. Ranamuka, A. P. Agalgaonkar, and K. M. Muttaqi, "Investigating the Operation of Multiple Voltage Regulators and DG in a Distribution Feeder,” Energy Procedia, vol. 14, pp. 1945-1950, Mar. 2012.

[3] D. Ranamuka, A. P. Agalgaonkar, and K. M. Muttaqi, "Dynamic Adjustment of OLTC Parameters using Voltage Sensitivity while utilizing DG for Volt/VAr Support,” in Proc. 2014 IEEE/PES General Meeting and Conference, pp. 1-8.

[4] D. Ranamuka, A. P. Agalgaonkar, and K. M. Muttaqi, "Mitigating Tapchanger Limit Cycles in Modern Electricity Networks Embedded with 
Local Generation Units,” in Proc. 2014 IEEE/IAS Annual Meeting and Conference., pp. 1-8.

[5] M. Basler, and R. C. Schaefer, "Understanding Power System Stability,” IEEE Trans. Industry Applications, vol. 44, no. 2, pp. 463474, Mar. 2008.

[6] C. M. Ong, Dynamic Simulation of Electric Machinery Using MATLAB-SimuLink, vol. 1. New Jersey: Prentice-Hall, 1998.

[7] P. Kundur, Power System Stability and Control, vol. 1. New York: McGraw-Hill, 1994.

[8] W. Freitas, J. C. M. Vieira, A. Morelato, and W. Xu, "Influence of Excitation System Control Modes on the Allowable Penetration Level of Distributed Synchronous Generators," IEEE Trans. Energy Conversion, vol. 20, no. 2, pp. 474-480, Jun. 2005.

[9] W. B. Gish, "Small Induction Generator and Synchronous Generator Constants for DSG Isolation Studies," IEEE Trans. Power Systems, vol. 1, no. 2, pp. 231-480, Apr. 1986.

[10] "SimuLink User Guide", [Online]. Available: http:// www.mathworks.com. 